\title{
Fatty Acid Patterns in the Classification of some Representatives of the Families Enterobacteriaceae and Vibrionaceae
}

\author{
By B. BØE AND J. GJERDE \\ Central Laboratory, Directorate of Fisheries, $N$-5000 Bergen, Norway
}

(Received 3 July 1979)

\begin{abstract}
Twenty-three strains representing the families Enterobacteriaceae and Vibrionaceae were analysed for fatty acid composition of whole cells by means of glass capillary column gas chromatography. Among the several alternatives tested, cluster analysis based on data normalized to hexadecanoate and logarithmically transformed provided good separations of species, genera and families. Strains from the genera Salmonella, Escherichia, Proteus, Enterobacter, Klebsiella, Vibrio and Aeromonas were studied.
\end{abstract}

\section{INTRODUCTION}

In recent years gas chromatography has been applied to the analysis of microbial cells and metabolic products (Mitruka, 1975). This technique can provide a detailed description of bacteria, since the analytical parameters are variables measured on a continuous scale. In this respect gas chromatography is superior to biochemical analyses which mainly produce binary data.

Identification of Gram-negative bacteria from aquatic and food sources has proved difficult due to the unsatisfactory state of their classification. Analyses of cellular fatty acids were therefore undertaken as a means of classifying various representative strains from the families Enterobacteriaceae and Vibrionaceae.

To extract the maximum useful information from the complex fatty acid profiles, mathematical techniques must be applied; various multivariate statistical methods exist for the analysis of such data (Sneath \& Sokal, 1973; Späth, 1977). In the present work, several algorithms were compared and it was found that cluster analysis based on a logarithmic transformation of data for fatty acid methyl esters (Jantzen et al., 1974) provided the best results.

\section{METHODS}

Bacterial cultures. Organisms used were: Escherichia coli NCTC 10082; Proteus morganii NCTC 2818 and NCTC 2815; Vibrio parahaemolyticus ATCC 17802; Enterobacter clocicae 20894/73, from the Culture Collection, Department of Microbiology, University of Bergen, Norway; Vibrio ichtyodermis (now called $V$. anguillarum), $V$. anguillarum V962, V767, V768, V873 and A272 and Aeromonas salmonicida A367/76 and A567/76, from the Culture Collection, National Veterinary Institute, Oslo, Norway; Salmonella typhimurium FSK1, Salmonella dublin FSK2, Enterobacter cloacae FSK20, Klebsiella pneumoniae FSK25, Vibrio anguillarum FSK14, FSK30, FSK31 and FSK45, Vibrio parcihaemalyticus FSK43 and FSK44, isolated at the Directorate of Fisheries, Central Laboratory, Bergen, Norway, and identified using the appropriate keys and diagnostic tables of Cowan et al. (1974).

Growth of bacteria and derivative formation. All cultures were grown on Plate Count Agar (Difco no. 0479 01 ) and incubated for 18 to $24 \mathrm{~h} ; \mathrm{V}$. anguillarum and $\mathrm{A}$. salmonicida were grown at $25^{\circ} \mathrm{C}$, and the other strains at $37^{\circ} \mathrm{C}$. For vibrio cultures the medium was supplemented with $\mathrm{NaCl}(2 \%, \mathrm{w} / \mathrm{v})$.

After incubation, cells were carefully removed from the plate surface and transferred to a test tube containing $5 \mathrm{ml}$ of $5 \%(\mathrm{w} / \mathrm{v}) \mathrm{NaOH}$ in $50 \%(\mathrm{v} / \mathrm{v})$ aqueous methanol. The fatty acids liberated by saponification were methylated with $\mathrm{BCl}_{3} /$ methanol, according to Moss et al. (1974). 
Gas-liquid chromatography (g.l.c.). A Perkin-Elmer F22 gas chromatograph equipped with a flame ionization detector and $25 \mathrm{~m} \times 0.25 \mathrm{~mm}$ i.d. glass capillary column coated with OV-101 (Perkin-Elmer) was used, with the injector at $230{ }^{\circ} \mathrm{C}$, the detector at $270{ }^{\circ} \mathrm{C}$, and the oven held at $150{ }^{\circ} \mathrm{C}$ for 2 min and then increased by $4{ }^{\circ} \mathrm{C} \mathrm{min}-1$ to $225^{\circ} \mathrm{C}$. Samples $(1 \mu \mathrm{l})$ were injected on the column by the splitless technique (Grob \& Grob, 1972, 1974). Results were quantified by electronic integration (Srectra Physics SP 4000 data system). Tentative identifications were established by retention times relative to methyl hexadecanoate, compared with a standard mixture, containing 23 fatty acid methyl esters (FAMEs), obtained from Supelco Inc. (no. 4-5436).

G.l.c./mass spectrometry. Mass spectra were obtained using an HP 5992 g.l.c./mass spectrometer equipped with a $27 \mathrm{~m} \times 0.25 \mathrm{~mm}$ i.d. OV-101 glass column. Spectra of FAMEs were compared with spectra of the 23 reference compounds. For FAMEs that could not be identified as belonging to the set of reference compounds, structures were tentatively assigned by comparing their mass spectra with those given by Ryhage $\&$ Stenhagen (1960).

Numerical analysis. The data were evaluated by a multivariate technique based on relative peak areas for 17 FAMEs measured for each of the bacteria. The necessary programs were written for a Hewlett Packard 9825 desktop calculator having a $32 \mathrm{~K}$ RAM memory to perform the following general functions: raw data input and output including editing; transformation of raw data; calculation of similarity coefficients; and cluster analysis. Four data sets were used.

Set no. 0: g.l.c. raw data normalized to the peak area for hexadecanoate. The complete raw data set consisting of relative peak areas for 17 FAMEs was used (Table 1) and, in addition, reduced sets consisting of only those FAMEs having relative peak areas $>10 \%$ were employed.

Set no. 1 : set 0 transformed according to $x=1+9\left(x-x_{\min }\right) /\left(x_{\max }-x_{\min }\right)$.

Set no. 2: set 0 logarithmically transformed, $x=\ln (1+x)$.

Set no. 3: set 0 autoscaled, $x=\left(x-x_{\mathrm{m}}\right) / S_{\mathrm{x}}$, where $x_{\mathrm{m}}$ is the mean value and $S_{\mathrm{x}}$ the standard deviation (N weighting).

Two similarity coefficients were used for these data sets, namely, the correlation coefficient R (Sokal \& Michener, 1958) and the coefficient based on euclidean distance $\mathrm{D}$ between pairs of bacteria, $1-\mathrm{D} / \mathrm{D}_{\max }$ (Harper et al., 1977). Clustering of strains was achieved by the unweighted pair group method (Sokal \& Michener, 1958; Sneath \& Sokal, 1973) and results are presented graphically as phenograms.

\section{RESULTS AND DISCUSSION}

Methyl esters of fatty acids containing from 12 to 20 carbon atoms were identified in the extracts of whole bacteria. A typical chromatogram is shown in Fig. 1. Up to 15 FAMEs were identified in each extract by comparing their mass spectra and relative retention times with those of the standards. The branched $\mathrm{C}_{16: 0}$ and $\mathrm{C}_{18: 0}$ acids were also included in the construction of the classification although standards were not available (Table 1). Compounds eluting earlier than $\mathrm{C}_{12: 0}$ were not studied further. Hydroxy acids were detected but were not included in the construction of the classification because of poor reproducibility of the relative peak areas. Two acids whose identity was only tentatively assigned, namely, branched $\mathrm{C}_{14: 0}$ and $\mathrm{C}_{14: 1}$, and which were found only in $V$. parahaemolyticus species were also not studied further.

In addition to the FAMEs described above, each chromatogram showed 10 to 20 unidentified components in the 1 to $10 \%$ relative peak area range. Analysis of the extracts from 10 subcultures of $V$. anguillarum FSK 14 revealed that eight of these small components showed good reproducibility and therefore provide data of value for classification. However, since none of these components were identified as fatty acid methyl esters, they were not included in the construction of the classification. The classification was based on only one type of compound in order to minimize problems of poor reproducibility caused by changing experimental parameters (e.g. g.l.c. columns).

By expressing the peak areas as percentages relative to hexadecanoate (Table 1) rather than to the total fatty acid methyl esters, interclass discrimination is favoured while intraclass relations are very little affected. 


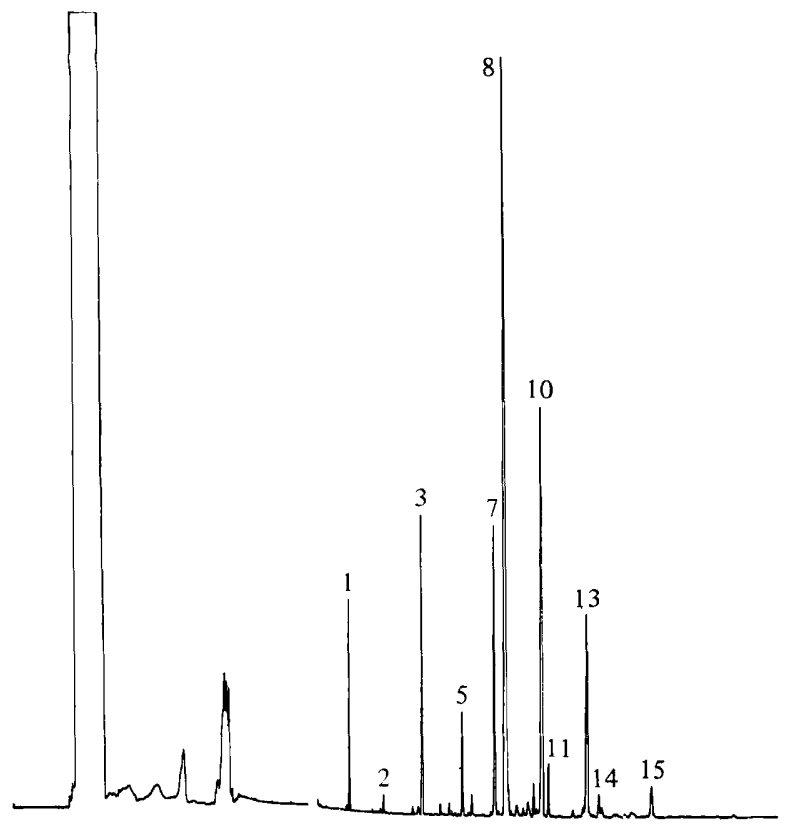

Fig. 1. Gas chromatogram of fatty acid methyl esters from $E$. coli NCTC 10082. The FAME designations corresponding to the numbers on the chromatogram are given in Table 1.

Few studies have been reported on the analysis of fatty acids isolated from bacteria belonging to the families Enterobacteriaceae and Vibrionaceae. The profile of the major fatty acids of $E$. coli was described by Ingram (1977), and was similar to that found in the present study. Distinguishing features of the family Enterobacteriaceae are the prominent hexadecanoate $\left(C_{16: 0}\right)$ peak, and the presence of hexadecenoate $\left(C_{16: 1}\right)$ and DL-cis-9,10methylene hexadecanoate $\left(\mathrm{C}_{17: 0 \Delta}\right)$ in comparable amounts at approximately $40 \%$ of the hexadecanoate peak. These characteristics have been reported previously for other members of the family Enterobacteriaceae (Machtiger \& O'Leary, 1973). Fatty acids isolated from $V$. cholerae were described by Brian \& Gardner (1968) who found a fatty acid pattern similar to that obtained from strains of Vibrio listed in Table 1.

The reproducibility of the FAME pattern was studied by analyses of 10 subcultures of $V$. anguillarum FSK14 (Table 2). Although the overall pattern was reproducible, the results varied for individual FAMEs, notably for those having relative peak areas below $10 \%$. For this reason and other reasons discussed below, we decided to study the behaviour of several algorithms applied to the full data set of 17 FAMEs, and also to sets comprising only FAMEs of relative peak area $>10 \%$.

The similarity matrix obtained by using algorithm R2 with 11 FAMEs, i.e. by using the logarithmic transformation 2 combined with correlation coefficients $R$, is shown in Table 3 . For each of the 10 subcultures of $V$. anguillarum, the maximum value of the similarity index was found, and the difference between the highest and lowest pairs was obtained. It can be seen from Table 3 that the highest pair is 1 and 7 , with $R=100$, and the lowest pair is 1 and 9 , with $\mathrm{R}=97$. The complete results for the eight algorithms used with 17 and 11 FAMEs are given in Table 4. Irrespective of the number of FAMEs included in the analysis, the raw data combined with correlation coefficients provide a treatment which is insensitive to variations in FAME pattern. Thus, this treatment cannot be expected to detect small but significant differences between bacteria. The algorithm R2 provides differences between pairs of bacteria of 6 and $3 \%$ for 17 and 11 FAMEs, respectively. These differences are considered to be appropriate considering that the test set consists of subcultures of one 


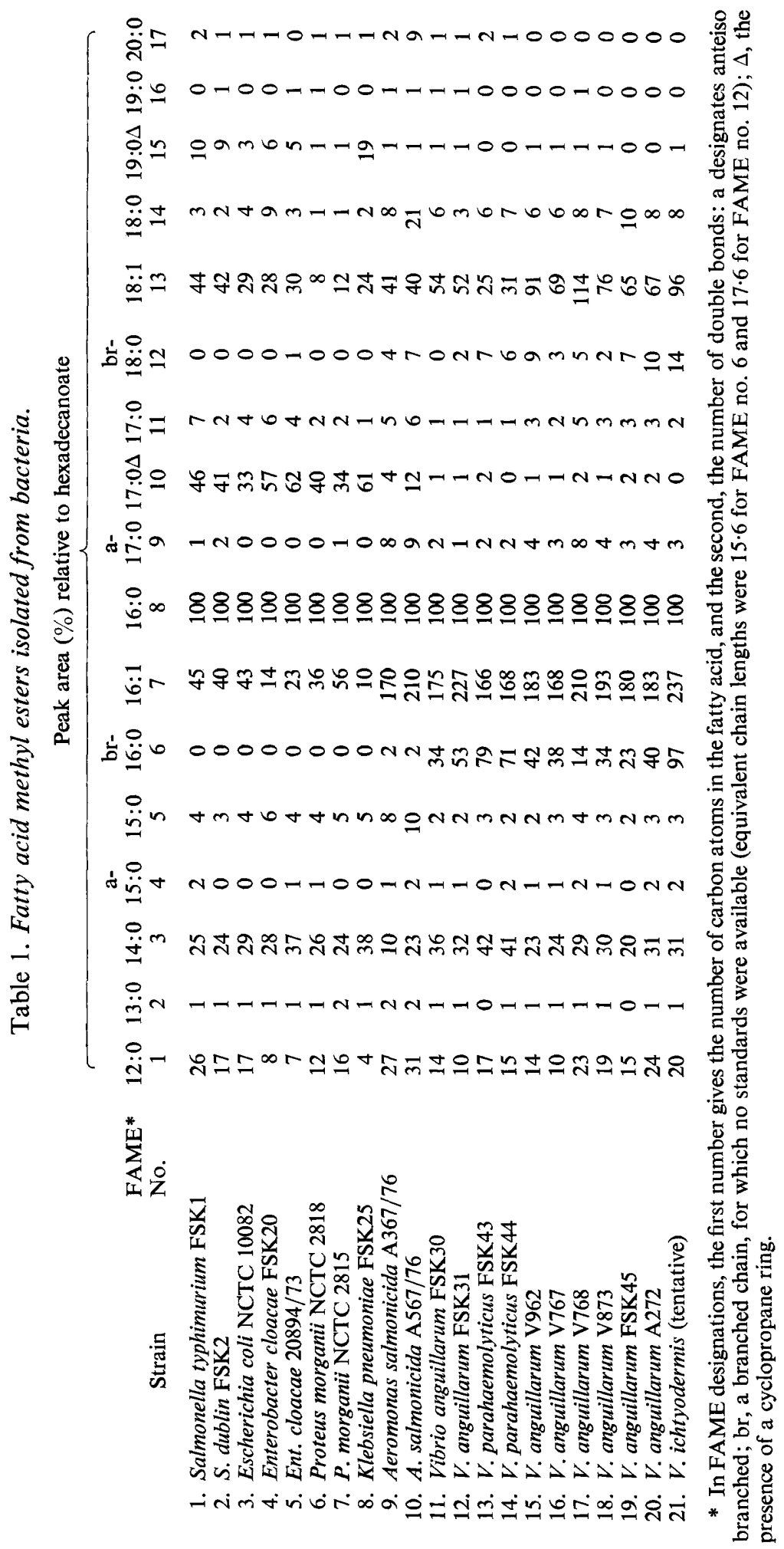




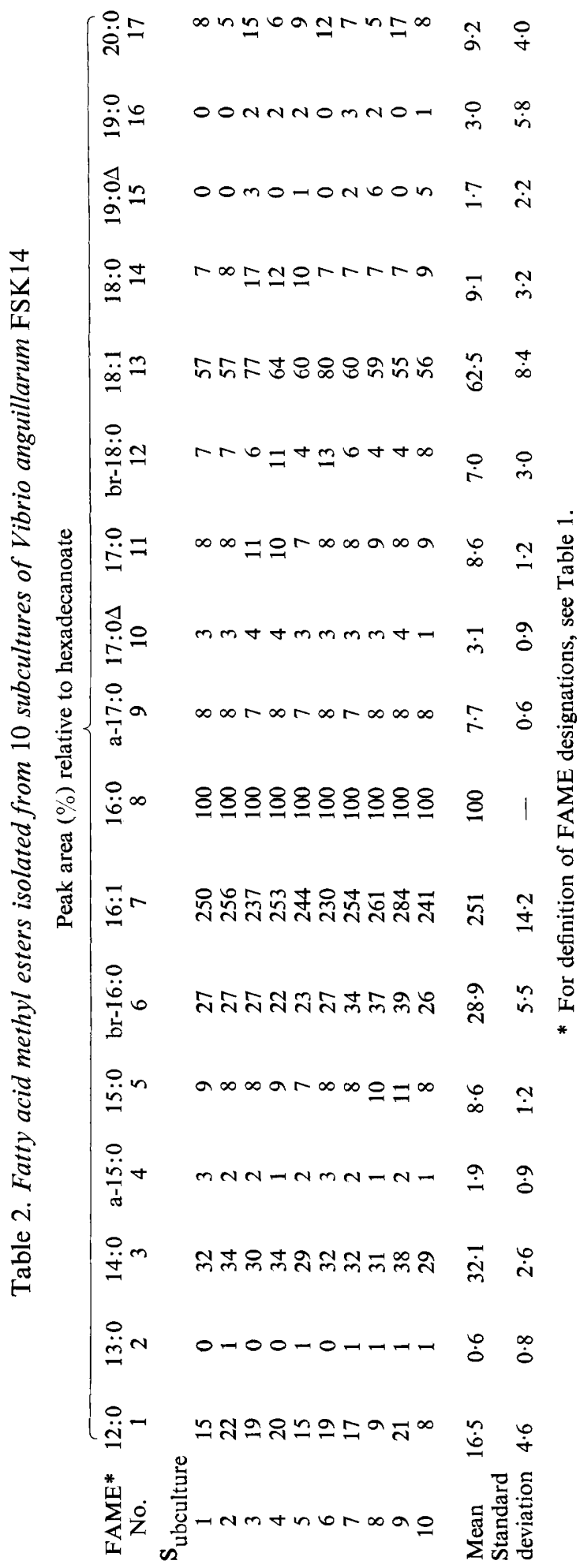


Table 3. Similarity matrix for 10 subcultures of Vibrio anguillarum FSK14 based on 11 FAMEs and algorithm R2

Based on the data from Table 2, FAMEs 2, 4, 9, 10, 15 and 16 being excluded. Correlation coefficients $\mathrm{R}(\times 100)$; raw data transformed according to $x=\ln (1+x)$.

$\begin{array}{cccccccccc}\begin{array}{c}\text { Sub- } \\ \text { culture }\end{array} & \mathbf{1} & \mathbf{2} & \mathbf{3} & 4 & 5 & 6 & 7 & \mathbf{8} & \mathbf{9} \\ 2 & 99 & & & & & & & & \\ 3 & 97 & 95 & & & & & & \\ 4 & 98 & 99 & 95 & & & & & \\ 5 & 99 & 98 & 99 & 97 & & & & \\ 6 & 98 & 97 & 95 & 97 & 96 & & & \\ 7 & 100 & 99 & 97 & 98 & 99 & 98 & & \\ 8 & 98 & 97 & 95 & 96 & 97 & 94 & 98 & \\ 9 & 97 & 95 & 96 & 92 & 97 & 94 & 97 & 95 & \\ 10 & 98 & 96 & 96 & 97 & 97 & 97 & 98 & 98 & 94\end{array}$

Table 4. Minimum and maximum values of similarity indexes $(\times 100)$ for pairs in the test set of 10 subcultures from Vibrio anguillarum FSK14

\begin{tabular}{|c|c|c|c|c|c|c|c|c|}
\hline \multirow[b]{2}{*}{ Transformation* } & \multirow[b]{2}{*}{ Similarity index } & \multirow[b]{2}{*}{ Code } & \multicolumn{3}{|c|}{ Using 17 FAME } & \multicolumn{3}{|c|}{ Using 11 FAME } \\
\hline & & & Highest & Lowest & $\begin{array}{c}\text { Differ- } \\
\text { ence }\end{array}$ & Highest & Lowest & $\begin{array}{c}\text { Differ- } \\
\text { ence }\end{array}$ \\
\hline 0 & $\mathbf{R}$ & Ro & 100 & 100 & 0 & 100 & 100 & 0 \\
\hline 0 & $1-\mathrm{D} / \mathrm{D}_{\max }$ & D0 & 84 & 46 & 38 & 85 & 52 & 33 \\
\hline 1 & $\mathbf{R}$ & R1 & 81 & 33 & 48 & 86 & 46 & 40 \\
\hline 1 & $1-\mathrm{D} / \mathrm{D}_{\max }$ & D1 & 70 & 29 & 41 & 74 & 32 & 42 \\
\hline 2 & $\mathbf{R}$ & R2 & 99 & 93 & 6 & 100 & 97 & 3 \\
\hline 2 & $1-\mathrm{D} / \mathrm{D}_{\max }$ & D2 & 80 & 49 & 31 & 79 & 38 & 41 \\
\hline 3 & $\mathrm{R}$ & R3 & 89 & 29 & 60 & 82 & 49 & 33 \\
\hline 3 & $1-D / D_{\max }$ & D3 & 67 & 34 & 33 & 77 & 32 & 45 \\
\hline
\end{tabular}

* 0 , None; $1, x=1+9\left(x-x_{\min }\right) /\left(x_{\max }-x_{\min }\right) ; 2, x=\ln (1+x) ; 3, x=x-x_{\mathrm{m}} / S_{\mathrm{x}}$.

strain. Transformations 1 and 3 tend to exaggerate the differences between the FAME patterns and may therefore be of value in studying the fine details of the phenograms. However, other studies (described below) indicate that these two transformations are of limited value.

Cluster analysis based on algorithm R2 and FAMEs with relative peak areas $>10 \%$ combines data derived from the 10 analyses of $V$. anguillarum FSK14 into one tight cluster with $\mathrm{R}=0.973$. A similar cluster was found for 10 subcultures of $V$. parahaemolyticus ATCC $17802(\mathrm{R}=0.969)$. The long-term reproducibility of the g.l.c. analysis was evaluated by analyses of the standard mixture over a 12 month period using two different columns. Cluster analysis revealed one tight cluster with $R=0.969$ based on algorithm $R 2$ and 15 of the FAMEs listed in Table 1, numbers 6 and 12 being excluded. Thus the raw g.l.c. data are of sufficiently high quality to permit further mathematical treatment.

The families Enterobacteriaceae and Vibrionaceae were easily separated by their different FAME patterns. Visual examination of the eight similarity matrices D,R-0,3 based on 17 FAMEs revealed that, with one exception, the two families were clearly separated. A typical similarity matrix is given in Table 5 . Algorithm R3 combining the transformation $x=$ $\left(x-x_{\mathrm{m}}\right) / S_{\mathrm{x}}$ with correlation coefficients did not separate the two families. Transformation 3 weights all FAMEs equally and the poor result obtained for the two families may be connected with the great variance already noted for FAMEs with relative peak areas $<10 \%$. However, as shown below, even in the case of using only FAMEs with relative peak areas $>10 \%$, transformation 3 is unsatisfactory. 
Table 5. Similarity matrix for bacteria no. 1 to 21 (see Table 1) based on 17

FAMEs and algorithm R0

Correlation coefficients $\mathrm{R}(\times 100)$; values greater than 80 .

$\begin{array}{rrrrrrrrrrrrrrrrrrrrr}\text { No. } & 1 & 2 & 3 & 4 & 5 & 6 & 7 & 8 & 9 & 10 & 11 & 12 & 13 & 14 & 15 & 16 & 17 & 18 & 19 & 20 \\ 2 & 100 & & & & & & & & & & & & & & & & & & & \\ 3 & 98 & 99 & & & & & & & & & & & & & & & & & \\ 4 & 93 & 94 & 93 & & & & & & & & & & & & & & & & \\ 5 & 94 & 95 & 94 & 99 & & & & & & & & & & & & & & & \\ 6 & 94 & 95 & 98 & 94 & 95 & & & & & & & & & & & & & & \\ 7 & 94 & 95 & 98 & 0 & 0 & 98 & & & & & & & & & & & & & \\ 8 & 0 & 91 & 0 & 98 & 98 & 92 & 0 & & & & & & & & & & & & \\ 9 & 0 & 0 & 0 & 0 & 0 & 0 & 0 & 0 & & & & & & & & & & & \\ 10 & 0 & 0 & 0 & 0 & 0 & 0 & 0 & 0 & 99 & & & & & & & & & & \\ 11 & 0 & 0 & 0 & 0 & 0 & 0 & 0 & 0 & 97 & 96 & & & & & & & & & \\ 12 & 0 & 0 & 0 & 0 & 0 & 0 & 0 & 0 & 96 & 96 & 99 & & & & & & & & \\ 13 & 0 & 0 & 0 & 0 & 0 & 0 & 0 & 0 & 0 & 0 & 96 & 96 & & & & & & & \\ 14 & 0 & 0 & 0 & 0 & 0 & 0 & 0 & 0 & 91 & 91 & 97 & 98 & 100 & & & & & & & \\ 15 & 0 & 0 & 0 & 0 & 0 & 0 & 0 & 0 & 95 & 93 & 98 & 97 & 92 & 94 & & & & & & \\ 16 & 0 & 0 & 0 & 0 & 0 & 0 & 0 & 0 & 96 & 95 & 99 & 98 & 94 & 96 & 100 & & & & & \\ 17 & 0 & 0 & 0 & 0 & 0 & 0 & 0 & 0 & 95 & 94 & 96 & 95 & 0 & 0 & 99 & 98 & & & & \\ 18 & 0 & 0 & 0 & 0 & 0 & 0 & 0 & 0 & 97 & 96 & 99 & 98 & 93 & 95 & 98 & 100 & 98 & & & \\ 19 & 0 & 0 & 0 & 0 & 0 & 0 & 0 & 0 & 98 & 97 & 99 & 98 & 92 & 94 & 99 & 99 & 98 & 100 & & \\ 20 & 0 & 0 & 0 & 0 & 0 & 0 & 0 & 0 & 97 & 96 & 100 & 99 & 95 & 96 & 99 & 100 & 97 & 100 & 99 & \\ 21 & 0 & 0 & 0 & 0 & 0 & 0 & 0 & 0 & 90 & 0 & 96 & 98 & 95 & 96 & 98 & 97 & 94 & 97 & 96 & 97\end{array}$

Table 6. Levels of similarity coefficients for Enterobacteriaceae

\begin{tabular}{ccccc} 
Algorithm & $\overbrace{\begin{array}{c}\text { Between } \\
\text { species }\end{array}}^{\text {Based on 17 FAME }}$ & $\overbrace{\begin{array}{c}\text { Between } \\
\text { genera } \\
\text { species }\end{array}}^{\text {Based on 7 FAME* }}$ & $\begin{array}{c}\text { Between } \\
\text { genera }\end{array}$ \\
R0 & $99,98,99$ & 98,98 & $99,97,99$ & 97,97 \\
D0 & $78,64,74$ & 66,66 & $80,64,77$ & 66,68 \\
R1 & - & - & $93,78,89$ & 64,79 \\
D1 & - & - & $77,74,66$ & 66,54 \\
R2 & $96,96,97$ & 97,94 & $98,98,98$ & 95,84 \\
D2 & $46,46,58$ & 45,48 & $86,82,80$ & 65,57 \\
D3 & - & - & $75,73,69$ & 66,52 \\
\multicolumn{4}{r}{ Having relative peak areas $>10 \%}$.
\end{tabular}

Bacteria 1 to 8 (see Table 1) represent five different genera within the Enterobacteriaceae. A successful cluster analysis must be able to group together strains belonging to the same genus and must also connect the constituent genera at a lower level of similarity. Examination of the eight phenograms based on 17 FAMEs revealed that algorithms R0, D0, R2 and D2 showed the expected connections between species and genera. However, levels of similarity values for species and genera overlapped and none of the algorithms based on the 17 FAMEs was satisfactory (Table 6). Many examples of cluster analyses based on FAMEs ranging from below 1 to $100 \%$ are given in the literature. These studies should be regarded critically, and especially so as in the majority of studies no indications are given concerning the reproducibility of the FAME patterns. Classifications based on seven FAMEs with relative peak areas $>10 \%$ provided the correct relationships with the algorithms R2, D2 and D3 (Fig. 2).

Bacteria 9 to 21 represent two genera within the family Vibrionaceae, i.e. Vibrio and Aeromonas. Within the genus Vibrio, three species were represented, viz. V. parahaemolyticus, $V$. anguillarum and $V$. ichtyodermis. Four algorithms (R0, D0, R2 and D2) were used with the full data set of 17 FAMEs obtained with these strains. Examination of the corresponding phenograms revealed that R0 and D0 did not separate the two genera, but successful 


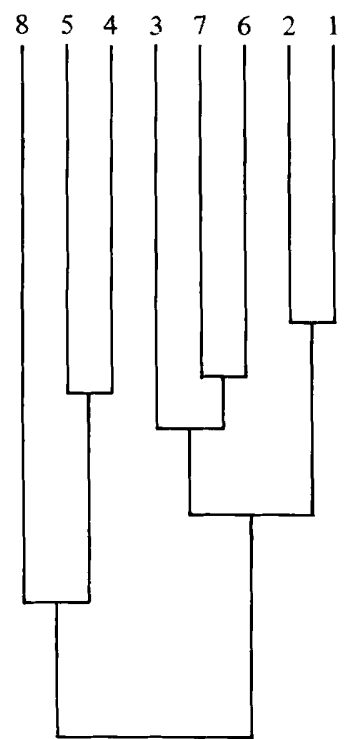

Algorithm D3

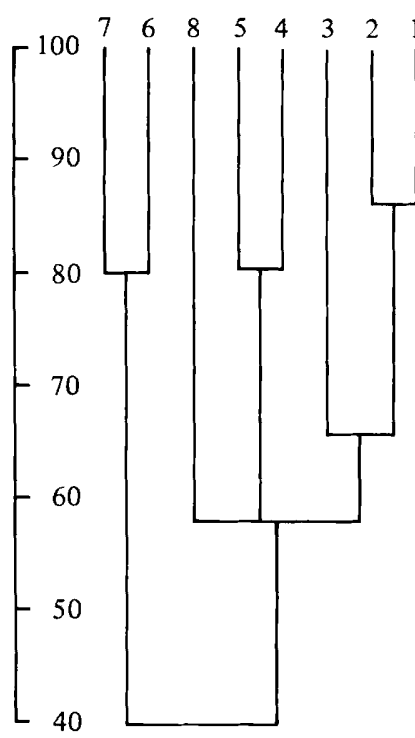

Algorithm D2
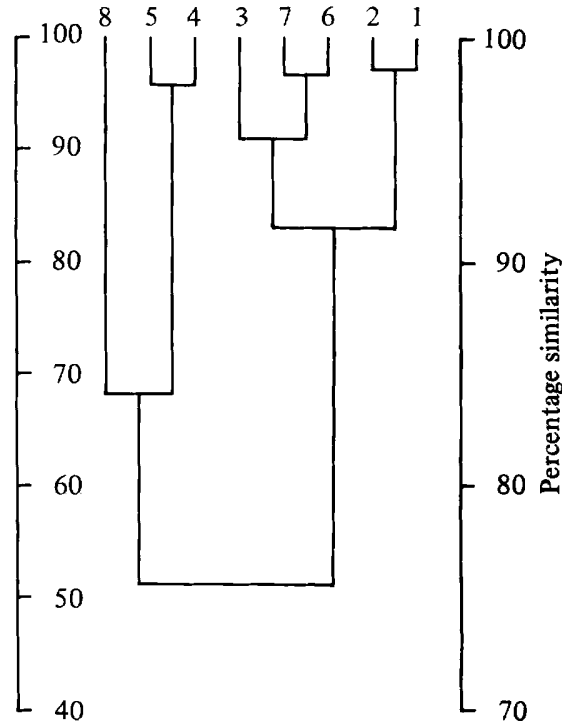

Algorithm R2

Fig. 2. Phenograms of Enterobacteriaceae strains based on seven fatty acid methyl esters and algorithms R2, D2 and D3.

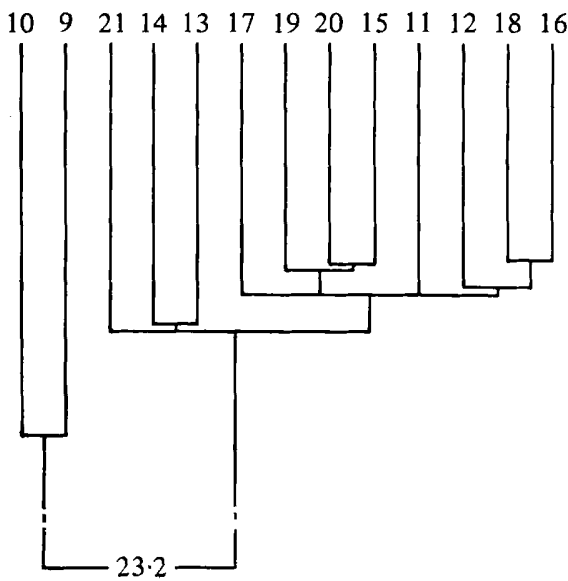

Algorithm D2

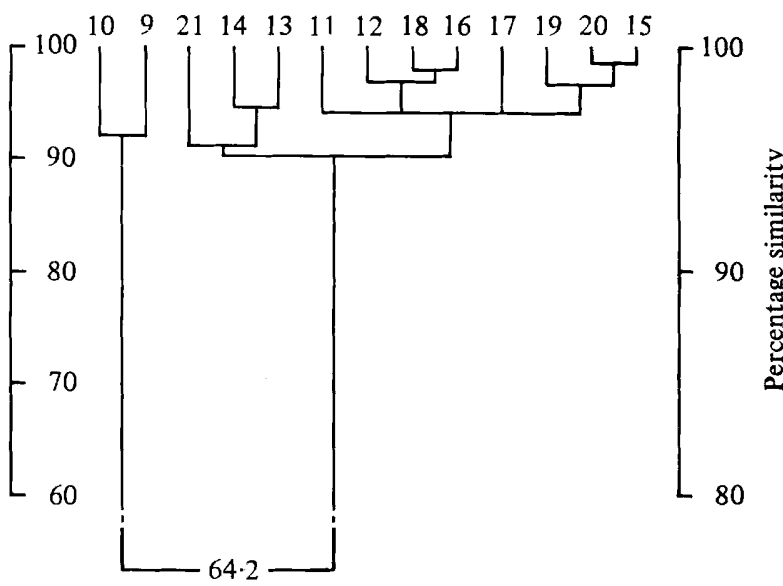

Algorithm R2

Fig. 3. Phenograms of Vibrionaceae strains based on 10 fatty acid methyl esters and algorithms R2 and D2.

separations were achieved using algorithms R2 and D2. However, the latter did not separate the three Vibrio species. Thus, we conclude that with the full data set, none of the algorithms worked well.

All eight algorithms R,D-0,3 were tested with a reduced data set comprising 10 FAMEs having relative peak areas $>10 \%$. The phenograms based on $\mathrm{R} 1, \mathrm{D} 1, \mathrm{R} 2$ and $\mathrm{D} 2$ gave the correct structure, while R0, D0, R3 and D3 did not separate the two genera. Comparison of the families Enterobacteriaceae and Vibrionaceae indicated that the algorithms R2 and D2 gave satisfactory results for both families when applied to reduced data sets consisting 
of FAMEs having relative peak areas $>10 \%$. Phenograms for bacteria 9 to 21 are given in Fig. 3.

Autoscaling (transformation 3) of data for FAMEs with relative peak areas $>10 \%$ did not give satisfactory results, irrespective of the type of similarity index used. Autoscaling is often used as a first step in data analysis, but the present results demonstrate that this transformation should be used with caution.

The following strategy for cluster analysis of bacteria is recommended as a result of the present study: families should be separated by examination of similarity matrices based on raw data for all FAMEs, with peak areas normalized to a prominent peak $\left(\mathrm{C}_{16: 0}\right)$, and the structure within families should be studied by using only FAMEs with relative peak areas $>10 \%$ and applying the logarithmic transformation $x=\ln (x+1)$ to the area normalized raw data.

\section{REFERENCES}

BriAN, B. L. \& GARdNER, E. W. (1968). Fatty acids from Vibrio cholerae lipids. Journal of Infectious Diseases 118, 47-53.

Cowan, S. T., Shewan, J. M. \& Veron, M. (1974). In Bergey's Manual of Determinative Bacteriology, 8 th edn, part 8 . Edited by R. E. Buchanan \& N. E. Gibbons. Baltimore: Williams \& Wilkins.

Grob, K. \& Grob, K., JR (1972). Methodik der Kapillar-Gaschromatographie. I. Teil: Die Direkteinspritzung. Chromatographia 5, 3-12.

Grob, K. \& Grob, G. (1974). Isothermal analysis on capillary columns without stream splitting. Journal of Chromatography 94, 53-64.

Harper, A. M., Duewer, D. L., Kowalski, B. R. \& FASchING, J. L. (1977). In Chemometrics, Theory and Application, pp. 14-52. Edited by B. R. Kowalski. Washington: American Chemical Society.

INGRAM, L. O. (1977). Changes in lipid composition of Escherichia coli resulting from growth with organic solvents and with food additives. Applied and Environmental Microbiology 33, 1233-1236.

J ANTZEN, E., Bergan, T. \& Bøvre, K. (1974). Gas chromatography of bacterial whole cell methanolysates. VI. Fatty acid composition of

strains within Micrococcaceae. Acta pathologica et microbiologica scandinavica B82, 785-798.

Machtiger, N. A. \& O'LeARY, W. M. (1973). Fatty acid compositions of paracolons: Arizona, Citrobacter, and Providencia. Journal of Bacterio$\log y$ 114, 80-85.

Mitruka, B. M. (1975). Gas Chromatographic Applications in Microbiology and Medicine. New York: John Wiley.

Moss, C. W., Lambert, M. A. \& Merwin, W. H. (1974). Comparison of rapid methods for analysis of bacterial fatty acids. Applied Microbiology 28, 80-85.

RyHaGe, R. \& STEnHagen, E. (1960). Mass spectrometry in lipid research. Journal of Lipid Research 1, 361-390.

SNeath, P. H. A. \& Sokal, R. R. (1973). Numerical Taxonomy. San Francisco: W. H. Freeman and Co.

SoKAl, R. R. \& Michener, C. D. (1958). A statistical method for evaluating systematic relationships. University of Kansas Science Bulletin 38, 14091438.

SрӓтH, H. (1977). Cluster-Analyse-Algorithmen. München: R. Oldenbourg Verlag. 